\title{
Abstracts of Award-Winning Posters, 15th Annual Health Sciences Poster Conference, Faculty of Medicine, Health Sciences Centre, Kuwait University, Kuwait, April 20-22, 2010
}

\section{Dr. Nael Al-Naqeeb Undergraduate Research Award}

\section{Best Postgraduate Awards}

\section{Basic Sciences (MSc)}

\section{Role of Pharmacists as Health Educators in Early Detection and Prevention of Breast Cancer in Kuwait \\ N.A. Abograis ${ }^{\text {a }}$,E.A. Abahussain ${ }^{\text {b }}$, M.G. Qaddoumi ${ }^{\text {a }}$ \\ Departments of applied Therapeutics and bPharmacy Practice, Faculty of Pharmacy, Kuwait University, Kuwait}

Introduction: Breast cancer is one of the commonest types of cancer among women worldwide. In Kuwait, it accounts for $36.3 \%$ of all types of cancer. This study was conducted to investigate the role of pharmacists and their willingness to educate patients about breast cancer risk and screening methods. Methods: An anonymous, non-experimental, cross-sectional questionnaire was designed and pharmacists' responses were collected using a self-administered questionnaire in 101 community and hospital pharmacists representing the Capital and Hawalli governorates of Kuwait. A knowledge score among pharmacists was deduced (weak, intermediate, and high) based on ability to answer correct questions. Multiple regression analysis and $\chi^{2}$ tests were used for data analysis. Results: $34 \%$ of the pharmacists displayed a low knowledge level about breast cancer prevention, whereas 56 and 9\% showed intermediate and high levels of knowledge, respectively. This level of knowledge was not significantly associated with gender, age, educational level, and work experience. Most of the pharmacists $(81 \%)$ were comfortable with discussing breast cancer education to patients but cited time and level of training as a hindrance. The majority of the pharmacists (83\%) were willing to educate female patients about breast cancer. Conclusions: This study highlights the importance for further investigations to evaluate the reasons behind the unsatisfactory knowledge level of breast cancer prevention among hospital and community pharmacists in Kuwait. Educational programs are needed to increase the level of knowledge such that pharmacists can assume an active role as health educators about breast cancer prevention in the future.

\section{Potential of TH-9, a Theophylline Derivative, as a Memory Enhancer in Dementia \\ H. Nashawi ${ }^{\text {a }}$, M.A. Oriowo ${ }^{\text {a }}$ S.B. Kombian ${ }^{\text {b }}$ \\ aDepartment of Pharmacology and Toxicology, Faculty of Medicine, and ${ }^{b}$ Department of Applied Therapeutics, Faculty of Pharmacy, Kuwait University, Kuwait}

Introduction: Dementia is a general term referring to cognitive deficit, including memory impairment. Alzheimer's disease (AD), the most common form of dementia, is one of the most disabling and burdensome health conditions worldwide. Memory loss, the main and initial complaint in $\mathrm{AD}$, is associated with defects in synaptic transmission and plasticity in the hippocampus and other brain areas. Since AD is largely an age-dependent disease and its prevalence continues to rise due to increasing human life expectancy, there is an urgent need for novel drugs that can cure AD. This study investigates the effect of $\mathrm{TH}-9$ on synaptic transmission, long-term potentiation (LTP) and long-term depression in young and old rats. Methods: $350-\mu \mathrm{m}$ coronal hippocampal slices were generated from brains of male Sprague-Dawley rats aged 1 and 20 months. Evoked, field excitatory postsynaptic potentials (fEPSPs) were recorded from the dendritic layer of area CA1 of the hippocampus by stimulating appropriate afferents. LTP was induced using high-frequency stimulation (HFS; $100 \mathrm{~Hz}$ for $1 \mathrm{~s}$ ) while LTD was elicited using low-frequency stimulation (LFS; $1 \mathrm{~Hz}$ for $5 \mathrm{~min})$. Results: TH-9 (10 $\mu \mathrm{M})$ increased the slopes of fEPSPs by $34.9 \pm 7.3 \%(\mathrm{p}<0.05)$ and $38.9 \pm 18.7 \%(\mathrm{p}<0.05)$ in young and old rats, respectively. LTP induction resulted in an increase of $59.9 \pm 11.0 \%(\mathrm{p}<0.05)$ and $47.4 \pm 16.5 \%(\mathrm{p}<0.05)$ in fEPSP slopes in slices from young and old rats, respectively. Induction of LTP in the presence of TH-9 resulted in a greater total increase in fEPSP slopes in old rats compared to young rats $(58.3 \pm 10.1 \%$ and

\section{KARGER}

Fax +4161306 1234 E-Mail karger@karger.ch www.karger.com 
$89.1 \pm 27.8 \%$, respectively). LFS depressed fEPSP slopes by $24.7 \pm$ $3.4 \%(\mathrm{p}<0.05)$ and $26.7 \pm 3.9 \%(\mathrm{p}<0.05)$ in young and old rats, respectively. However, pretreatment with TH-9 abolished LTD responses in old but not young rats. Conclusions: TH-9 enhances LTP in hippocampal slices of both young and old rats while preventing LTD maintenance only in older rats. This action of TH-9 is consistent with a potential to be used for dementia.

Funding Agency: Kuwait University Research Administration Grant No. YM08/08.

\section{Resident}

\section{Associations of Leptin, Leptin Receptor and Free Leptin Index with Metabolic Phenotypes of PCOS}

F.H.Safar ${ }^{\text {a }}$, O.A. Mojiminiyi ${ }^{a}$, H. Al Rumaih ${ }^{\text {b }}$, T. Al Rammah ${ }^{\text {a }}$, F.M.E. Diejomaoh ${ }^{\mathrm{a}}$

aFaculty of Medicine, Kuwait University, and ' IVF Unit, Maternity Hospital, Kuwait

Introduction: Polycystic ovaries syndrome (PCOS) is a complex endocrine disorder with heterogeneous presentation and different phenotypes in women of reproductive age. This study evaluates the association, if any, between circulating leptin, leptin receptor (sObR), free leptin index (FLI) with the metabolic phenotypes of PCOS. Methods: We measured follicular phase hormones, androgens, SHBG, fasting lipid profile, leptin, sObR, FLI, glucose, insulin and insulin sensitivity (\%S) and resistance using the homeostasis model assessment (HOMA-IR). Univariate and logistic regression analyses were used to find the associations of these variables with each other and with metabolic phenotypes of PCOS. Results: PCOS patients had significantly higher leptin $(p=0.03), F L I(p=0.02)$ and lower sObR $(p=0.03)$ than controls. Leptin $(\mathrm{p}=0.01)$ and FLI $(\mathrm{p}=0.01)$ were significantly higher in metabolic syndrome positive MS+ve PCOS patients while sObR was significantly lower $(\mathrm{p}=0.03)$. The levels of leptin $(\mathrm{p}=0.004)$ and FLI ( $\mathrm{p}=0.002)$ were also significantly higher while sObR levels were significantly lower $(\mathrm{p}=0.01)$ among PCOS women with HOMA-IR $\geq 2$. However, no significant differences were detected in leptin, sObR and FLI between hyperandrogenic and normoandrogenic PCOS. Leptin and sObR had significant direct correlations with waist, BMI, TG, insulin, HOMA-IR, FAI and inverse correlations with SHBG, \%S and HDL-C. sObR had similar but inverse significant correlations. Leptin (OR 1.04, $\mathrm{p}=0.003)$, sObR (OR $0.88, \mathrm{p}=0.03)$ and FLI (OR 1.32, $\mathrm{p}=0.01)$ were significantly associated with MS. Leptin (OR 1.05, $\mathrm{p}=0.001)$, sObR (OR 0.82, $\mathrm{p}=0.01)$ and FLI (OR 1.46, $\mathrm{p}=0.002)$ were also significantly associated with insulin resistance phenotypes. No significant associations were found with androgens. Conclusions: We conclude that leptin, sObR and FLI are significantly associated with metabolic and insulin resistance phenotypes of PCOS but leptin does not appear to contribute to the hyperandrogenic status in PCOS patients.

Funding Agency: Kuwait University Research Administration Grants MG 01/05 and YM 21/07.

\section{Basic and Applied Sciences Awards}

\author{
1 \\ (-)-Epigallocatechin-3-Gallate (EGCG) Promotes \\ Functional and Sensory Recovery in Crushed Rat \\ Sciatic Nerve
}

W.M. Renno ${ }^{\text {a }}$ M. Al-Maghrebi ${ }^{\text {b }}$, P. George ${ }^{\text {a }}$, A. Mathur ${ }^{\text {a }}$

Departments of anatomy and ${ }^{\mathrm{b}}$ Biochemistry, Faculty of

Medicine, Kuwait University, Kuwait

Introduction: (-)-Epigallocatechin-3-gallate (EGCG), the most abundant polyphenol in green tea (GT), has been shown to protect several brain functions. Recently, we have shown that GT consumption improves both reflexes and sensation which are often affected in the course of peripheral neuropathy in unilateral chronic constriction injury to the sciatic nerve. Considering the substantial neuroprotective properties of GT polyphenols, we sought to investigate whether EGCG could improve motor and sensory impairments induced by crushing the sciatic nerve. Methods: Wistar male rats $(n=8)$ were randomly assigned to three groups as follows: control sham group, experimental control group with sciatic nerve crush treated with saline (i.p.) and experimental group with sciatic nerve crush treated with $50 \mathrm{mg} / \mathrm{ml}$ EGCG (i.p.) (1-2 h, 1 day and 2 days after the crush injury). The duration and magnitude of functional, thermal and behavioral hyperalgesia recovery were monitored. Proproiceptive integrity was evaluated by assessing the response to tactile placing and the hopping response. Extensor postural thrust (EPT) was assessed on a digital balance. Results: EGCG-treated axonotomized group showed significant improvement in the toe spread and foot positioning analysis compared to the experimental control group. Moreover, EGCG treatment resulted in an earlier and significant gain of hindlimb extension force as evaluated by the EPT and derived percentage motor deficit. Likewise, the proprioceptive and motor function expressed by the hopping response was fully restored 2-3 weeks earlier in EGCG treated group compared to saline treated controls. In addition, EGCG significantly reduced the axonotmesis-induced thermal and mechanical hyperalgesia as well as mechanical allodynia compared to saline treated animals. Conclusions: Our results demonstrate that EGCG enhances functional and sensory recovery of peripheral nerve injuries in rat and thus improves nerve regeneration.

2

PROPRED Analysis for Prediction of HLA-

DR-Promiscuous Regions and Epitopes of Mycobacterium tuberculosis Protein Rv1980c, and Their Recognition by Human T Cell Lines

A.S. Mustafa, F. Shaban

Department of Microbiology, Faculty of Medicine, Kuwait University, Kuwait

Introduction: Rv1980c, a major secreted antigenic protein of Mycobacterium tuberculosis, is encoded by M. tuberculosis-specific region of difference 2 , which is deleted in many vaccine 
strains of BCG. Thus, this protein could be useful in the diagnosis of tuberculosis (TB) as well as a new vaccine against TB. However, to have world-wide application, this protein should have HLApromiscuous regions/epitopes recognized by human T helper-1 (Th1) cells. The aim of this study was to determine HLA-promiscuous regions/epitopes of Rv1980c using a computational method, and confirm the prediction results in Th1 cell assays. Methods: The sequence of Rv1980c protein was analyzed for binding to 51 HLA-DR alleles using PROPRED, a computational method. The prediction results were experimentally verified by testing 20 mer synthetic peptides corresponding to the predicted HLA-DRbinding regions and epitopes with $\mathrm{T}$ cell lines established from peripheral blood of PPD-positive and HLA-heterogeneous healthy subjects in Th1-cell assays (antigen-induced proliferation and interferon- $\gamma$ secretion). Results: The PROPRED analysis suggested that Rv1980c sequence could bind to all 51 HLA-DR alleles. Furthermore, PROPRED identified 26 epitopes and eight nonoverlapping HLA-DR-binding regions (9-35 aa in length) in Rv1980c sequence, with five regions (aa $20-44$, aa 68-102, aa $132-146$, aa 164-186 and aa 194-202) being HLA-DR promiscuous. The results of testing synthetic peptides with T cell lines in Th1 cell assays showed that four peptides belonging to four HLA-promiscuous regions of Rv1980c (aa 21-40, aa 81-100, aa 171-190 and aa 191-20), had immunodominant epitopes and were HLA-DR promiscuous. Conclusions: The computational method (PROPRED) used in this study suggested promiscuous HLA-DR binding of mature MPT64 (Rv1980c) sequence. In addition, PROPRED could faithfully identify four HLA-promiscuous and immunodominant regions/epitopes of Rv1980c.

Funding Agency: Kuwait University Research Administration Grant No. MI03/05.

\section{Clinical Sciences Awards}

\author{
1 \\ Schizophrenia Psychopathology in a Kuwaiti Arab
Sample \\ M.A. Zahid ${ }^{\mathrm{a}}$, J.U. Ohaeri ${ }^{\mathrm{b}}$ \\ aDepartment of Psychiatry, Faculty of Medicine, Kuwait \\ University, and ${ }^{\mathrm{b}}$ Department of Psychiatry, Psychological \\ Medicine Hospital, Kuwait
}

Introduction: The heterogeneity of schizophrenia psychopathology has led to a search for symptom clusters that could be related to broad features of the disease. The objectives of the study were to: (1) highlight the pattern of symptoms among Kuwaiti subjects with schizophrenia, using the ICD-10 symptom checklist, (2) assess the factor structure of the BPRS-18 and BPRS-24, and (3) assess the relationship of the resulting factors with sociodemographic characteristics, age at onset of illness, family history of mental illness, indices of quality of care and psychological well-being. Methods: Consecutive outpatients in stable condition were assessed with the Brief Psychiatric Rating Scale and ICD-10 Symptom Checklist, as well as measures of quality of care. Results: There were 130 patients $(66.1 \%$ males, mean age 36.8 , duration of illness 12.9 years). Of the ICD-10 symptoms, the commonest positive symptoms were hallucinations (58.5\%) and delusions
(72\%). Catatonic symptoms were rare (2.9\%). About one-quarter of subjects experienced four of the negative symptoms. In exploratory factor analysis, we broadly replicated the known syndromes for BPRS-24 (disorganization, positive, activation, manic, negative and depression) and BPRS-18 (negative, positive, activation and affect). In regression analyses, the variables independently associated with psychopathology were family income, negative affect, self-esteem, duration of illness, age, lack of money for enjoyment, met needs for care, and caregiver tension. The negative syndrome had more significant associations with the variables investigated than the positive syndrome. Psychopathological scores were separable from indices of psychological well being. Conclusions: In this first study of schizophrenia psychopathology from the Arab world, the clinical manifestations were similar to the data from the developed countries. The persistence of psychotic symptoms despite freely available antipsychotic treatment, and the impact on caregiver burden, call for attention to the perennial issue of treatment resistance, and underscore the need for continued interaction with family members.

Funding Agency: Kuwait University Research Administration Grant No. MQ01/05.

2

Large-Scale Evaluation of Multiplex PCR and DNA Sequencing of Internal Transcribed Spacer Region for Rapid Differentiation and Identification of Mycobacterium Species Isolates in Kuwait

E. Mokaddas, S. Ahmad

Department of Microbiology, Faculty of Medicine, Kuwait University, Kuwait

Introduction/Objective: Specific identification is of clinical relevance since treatment varies according to Mycobacterium species causing infection. This large-scale study evaluated the performance of a multiplex PCR (mPCR) assay for rapid differentiation of acid-fast bacilli (AFB)-positive liquid cultures as Mycobacterium tuberculosis (MTB) or nontuberculous mycobacteria (NTM) and DNA sequencing of the 16S-23S internal transcribed spacer (ITS) region to confirm each NTM isolate. Methods: A total of 993 AFBpositive cultures by the MGIT 960 system in Kuwait were used. The DNA was extracted by the boiling method and MPCR targeting oxyR-ahpC intergenic region and $\mathrm{rpoB}$ gene was performed to differentiate NTM from MTB. The 16S-23S ITS region was amplified and sequenced by using pan-mycobacterial primers. Mixed cultures were identified by a line probe assay. Results: The mPCR identified 924 isolates as MTB, 67 isolates as NTM and two isolates as mixed cultures. Thirteen different NTM species were identified. Of the 67 NTM isolates, 19, 12, 10, 8, 6, 2, 2 and 2 isolates were identified as $M$. kansasii, $M$. fortuitum, $M$. abscessus, $M$ avium, $M$. intracellulare, M. lentiflavum, M. gordonae, and M. chelonae, respectively. One isolate each was identified as M. chimaera, $M$. parascrofulaceum and $M$. immunogenum while two isolates were identified as Mycobacterium species. One NTM isolate contained a mixed culture, M. kansasii and M. scrofulaceum. The 150 randomly selected MTB isolates were identified as M. tuberculosis by 16S-23S ITS sequencing and/or hybridization with MTB-specific probes. Both mixed cultures contained M. tuberculosis and M. fortuitum. Conclusions: The mPCR accurately and rapidly differenti- 
ated all MTB from NTM isolates and DNA sequencing of $16 \mathrm{~S}-23 \mathrm{~S}$ ITS region identified nearly all NTM isolates. Rapid differentiation as MTB or NTM by mPCR followed by species-specific identification of NTM by DNA sequencing is most suitable for proper management of mycobacterial infections in Kuwait.

Funding Agency: Kuwait University Research Administration Grant No MI02/04.

\section{3}

\section{Folate, Vitamin $B_{12}$ and Homocysteine Levels in Kuwait Adolescent Subjects: Potential Implications for Cardiovascular Disease Risk in Later Life}

\section{A.O. Akanji ${ }^{\text {a }}$ L. Thalib ${ }^{\text {b }}$, A.N. Al-Isa ${ }^{\text {b }}$ \\ Departments of aPathology and ${ }^{\mathrm{b}} \mathrm{Community}$ Medicine, Faculty of Medicine, Kuwait University, Kuwait}

Introduction: Elevated circulating fasting total homocysteine (tHcy) concentration is associated with an increased risk of occlusive vascular disease in adults. Important determinants of tHcy levels are folate and vitamin $B_{12}$. This study investigated age, gender, and body mass as determinants of folate, vitamin $\mathrm{B}_{12}$ and tHcy levels in Kuwaiti Arab adolescents and to propose population, gender and age-specific reference ranges for these biomarkers. Methods: A total of 774 (316 boys, 458 girls) apparently healthy 10 - to 19 -year-olds attending various secondary schools in Kuwait were assessed for anthropometry, blood pressure and fasting blood levels of Hcy, folate and vitamin $\mathrm{B}_{12}$, after informed voluntary parental/guardian consent. Results: Boys had significantly higher waist/hip ratio, tHcy and folate concentration than girls, although BMI was similar for both groups. Vitamin $B_{12}$ was greater in the girls. In all, tHcy had positive correlations with both markers of adiposity (BMI and WHR) and with systolic blood pressure. Additionally, tHcy was negatively correlated with folate and vitamin $\mathrm{B}_{12}$. These relationships persisted on multiple regression. Folate and vitamin $B_{12}$ levels decreased significantly with age. Correspondingly, Hcy levels increased, with mean values $(\mu \mathrm{mol} / \mathrm{L})$ for boys $(6.6 ; 7.9)$ and girls $(4.5 ; 6.2)$ aged $10-14$ and $14-19$ years, respectively. Conclusions: There is an age-related increase in tHcy in adolescents reflecting decreased levels of folate and vitamin $B_{12}$. It is suggested that age-related reference ranges for $\mathrm{tHcy}$ and associated vitamins be used in adolescents, to assess and prevent future cardiovascular disease risk.

Funding Agency: KURA/KFAS Grant No. MC 01/04.

\section{Case Report Award}

\section{Scoliosis as a Rare Risk Factor for Colon Perforation during Colonoscopy: A Case Report}

O. AlHarbi, S. AlOsaimi, I. AlKandari

Department of Surgery, Farwanyia Hospital, Kuwait

Background: Colonoscopy has been used for the diagnosis and treatment of a wide range of diseases. A variety of complications are associated with colonoscopy, the most serious one is perforation of the colon, which is more frequent after therapeutic procedures. It is a rare but life-threatening complication and may require surgical intervention, or conservative treatment in selected cases. There are some risk factors associated with iatrogenic colonoscopic perforation (CP) such as diverticular disease and previous intra-abdominal surgery. Knowing the factors influencing $\mathrm{CP}$ is important to avoid or minimize it. We are describing an unreported risk factor for iatrogenic CP. Case Summary: A 59-year-old female underwent colonoscopy screening for chronic anemia. She was well prepared and the procedure went smoothly, with perfect tolerance and sedation. No abnormalities were detected up to the cecum, but the endoscopist noted a small tear in the sigmoid colon upon pulling the scope against her bony structures. A few hours later she developed fever and lower abdominal pain which gradually increased. Abdominal X-ray showed severe scoliotic deformity of the lumber spine with left convexity and pneumo-retroperitoneum. CT scan detected a thickened wall of the sigmoid colon with a mild amount of air and fluid collection in the pelvis, mainly perisigmoid in location. Clinically, she was stable, having tender lower abdomen. The case was successfully managed conservatively and follow-up CT scan showed reduction of that collection. The mechanism of $\mathrm{CP}$ in our case is related to the bony deformity which precipitated the injury. Conclusion: Patients with severe scoliosis and skeletal deformity undergoing colonoscopy, have a higher risk of $\mathrm{CP}$ as the scope is pushed/pulled against unexpectedly, abnormally located boney structures. Symptoms of abdominal pain or peritonitis following colonoscopy in this group of patients should be highly suspected for colon injury and should be managed accordingly. 